ENCYCLOPÉDIE Encyclopédie berbère

BERBERE

$30 \mid 2010$

30 | Maaziz - Matmata

\title{
Macota / Maghrawa (Tunisie)
}

\section{A. M'charek}

\section{OpenEdition}

Journals

Édition électronique

URL : http://journals.openedition.org/encyclopedieberbere/388

DOI : $10.4000 /$ encyclopedieberbere.388

ISSN : 2262-7197

\section{Éditeur}

Peeters Publishers

\section{Édition imprimée}

Date de publication : 29 décembre 2010

Pagination : 4465-4468

ISBN : 978-90-429-2367-6

ISSN : 1015-7344

\section{Référence électronique}

A. M'charek, « Macota / Maghrawa (Tunisie) », Encyclopédie berbère [En ligne], 30 | 2010, document

M07, mis en ligne le 17 septembre 2020, consulté le 18 octobre 2020. URL : http://

journals.openedition.org/encyclopedieberbere/388 ; DOI : https://doi.org/10.4000/ encyclopedieberbere.388

Ce document a été généré automatiquement le 18 octobre 2020.

(c) Tous droits réservés 


\title{
Macota / Maghrawa (Tunisie)
}

\author{
A. M'charek
}

\section{Le site archéologique}

1 Macota-Maghrawa se trouve à $8 \mathrm{~km}$ à vol d'oiseau au nord-ouest de Maktar*, dans une région montagneuse correspondant aux contreforts septentrionaux de la hamada des Ouled Ayar, fortement disséqués par l'érosion (Atlas Archéologique de la Tunisie, II, feuille de Maktar, 125, Maghraoua). Le site naturel est un éperon légèrement incliné vers le nord et merveilleusement exposé, encadré par deux ravins relativement encaissés. La localité moderne de Maghrawa, aujourd'hui simple «douar » comptant une trentaine de maisons paysannes, est bâtie sur les ruines d'une bourgade antique peu étendue, dont le noyau urbain ne devait pas dépasser une dizaine d'hectares environ.

2 Ayant servi de carrière, le site archéologique est en mauvais état de conservation. Quelques rares vestiges sont encore visibles au nord du champ de ruines alors que la partie sud de ce dernier, plus large et moins érodée, est actuellement aménagée par les paysans en jardins voués aux cultures maraîchères et irrigués par deux sources de bonne qualité, dont Aïn-Maghrawa à $300 \mathrm{~m}$ en amont du village. La principale nécropole se trouve à l'ouest sur la berge de l'oued el-Mislen où plusieurs dolmens sont encore debout.

3 Très visitées au XIX ${ }^{e}$ siècle par les voyageurs européens (Grenville Temple, Nathan Davis, Honegger, Willmans, Maltzan, Bart, Pelissier, Cagnat et Saladin), les ruines antiques de Maghrawa sont demeurées peu connues des archéologues contemporains, à l'exception des protohistoriens qui ont souvent accordé un intérêt particulier à ses monuments mégalithiques (Atlas Préhistorique de la Tunisie, f. de Maktar, 8, 1985, p. 27 et $\mathrm{n}^{\circ}$ 35). Le site servait alors de "nid d'aigle" à la tribu des Ouled Ayar : le caïd de cette tribu y était installé avec sa zmala et, ce n'est qu'avec le Protectorat français en 1881 que Maktar moderne prit le pas sur Maghrawa, en devenant à la fois le siège du contrôle civil et du caïdat.

Deux campagnes de fouilles empiriques ont été effectuées à Maghrawa au XIX ${ }^{\mathrm{e}}$ siècle : en 1842 , la première a permis à l'allemand Honegger de recueillir des inscriptions néo- 
puniques et latines ainsi qu'une série remarquable de reliefs numides qu'on a, plus tard, faussement attribuée à la Ghorfa ; la deuxième fouille, publiée en 1893, est due à C. Denis qui a trouvé dans deux dolmens de la localité un grand nombre de squelettes et une centaine de vases souvent recouverts d'un émail de couleur verte. Une prospection relativement récente a donné l'occasion à Mansour Ghaki de recueillir à Maghrawa et ses environs huit inscriptions libyques et quelques inscriptions néo-puniques. Le site avait depuis longtemps livré ce type de document: l'allemand Honegger y avait découvert en 1837 la deuxième inscription libyque qui ait été connue des savants. Pour notre part, nous avons trouvé à Maghrawa un nombre important de textes latins et de reliefs à la fois votifs et funéraires qui étaient le plus souvent remployés dans les maisons paysannes.

\section{Macota, centre des Micatanes et vicus de Mactaris}

Dans un article de mise au point (M'charek 1988), nous avons établi l'origine de la fameuse collection réunie au XIX siècle par l'Allemand Honegger et aujourd'hui dispersée entre les musées de Londres, Paris, Vienne, Le Bardo à Tunis et le site de Dougga.

TERRITOIRES VOISINS DE MACOTA ET MACTARIS

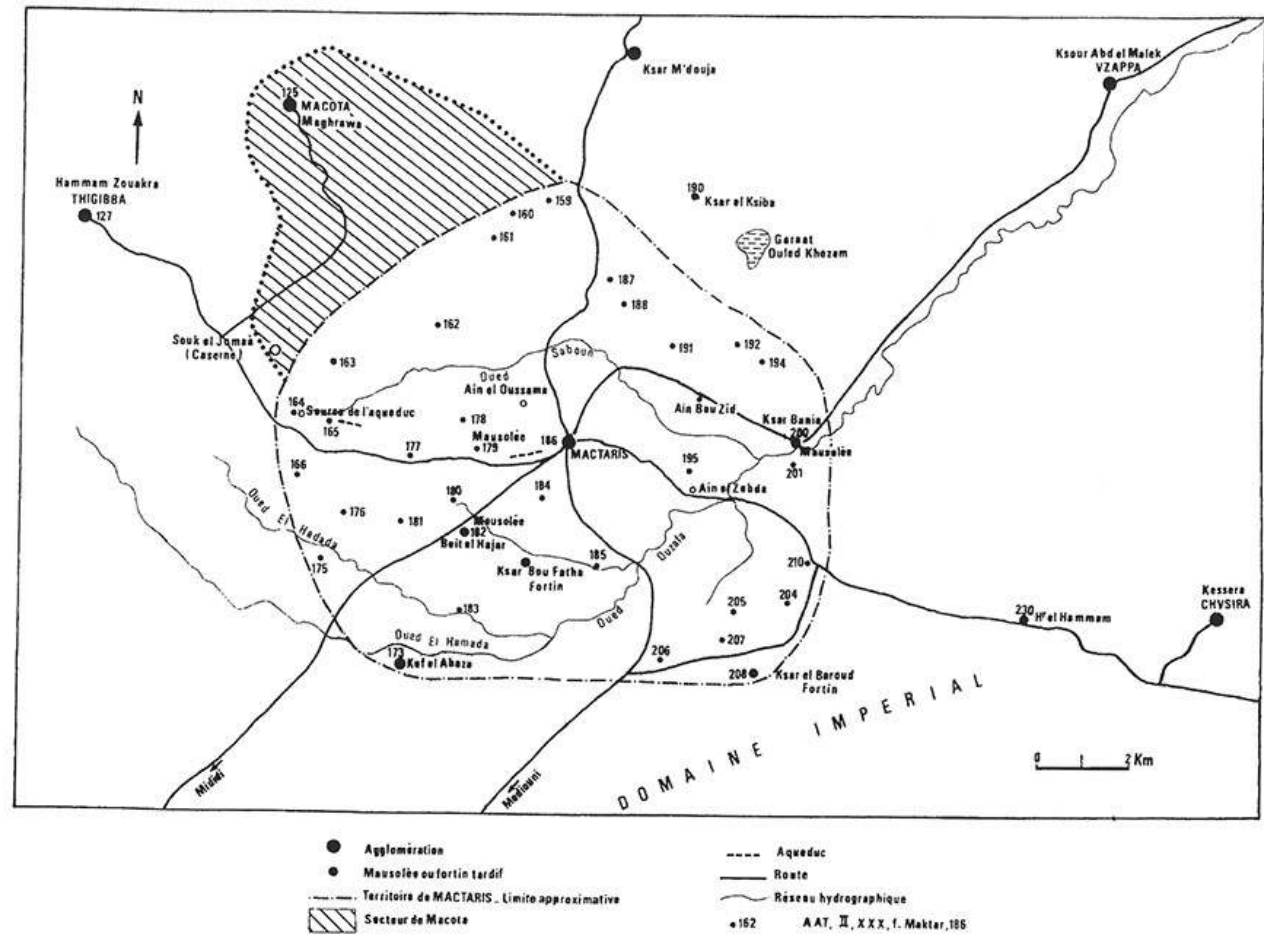

6 En même temps que ces reliefs votifs, Honegger a récupéré à Maghrawa une série importante de stèles funéraires épigraphes dont plusieurs comportaient un décor sculpté. Les inscriptions votives ou funéraires néo-puniques ou punico-latines, provenant de cette "collection Honegger" ont été, pour la plupart, publiées au milieu $\mathrm{du} \mathrm{XIX}^{\mathrm{e}}$ siècle et plusieurs fois commentées par les épigraphistes. Leur provenance exacte et le contexte de leur découverte étant connus depuis 1988, il était devenu possible de mieux les exploiter scientifiquement. C'est ainsi que le contenu de six inscriptions néo-puniques (deux votives à Baal Hammon et quatre funéraires provenant 
d'un tombeau familial), éclairé par l'apport des inscriptions latines et celui des sources littéraires, nous a paru suffisant pour :

- Identifier le nom antique de Maghrawa: $M(a) k(o) d(a)$ dans un texte néo-punique et Macota dans les listes épiscopales de la province de Byzacène ;

- Situer, hypothétiquement dans la région de Maktar, l'antique Thusca, le territoire des Micatanes, tribu numide mentionnée par Diodore de Sicile;

- Evaluer le centre d'une agglomération secondaire de la Thusca au I $\mathrm{I}^{\mathrm{er}}$ siècle ap. J.-C. : le centre urbain doté d'un murus publicus d'époque numide, était administrée par deux rab-s (= magistri) éponymes.

- Entrevoir les liens privilégiés entre Macota, communauté secondaire (vicus ou castellum) et sa voisine Mactaris, chef-lieu du pagus Thuscae regroupant, aux deux premiers siècles de l'ère chrétienne, une cinquantaine de cités.

\section{BIBLIOGRAPHIE}

BEJAOUI F. et GHAKI M., 1987 - « Maghraoua dans l'Antiquité », REPPAL, III, p. 255-259.

DENIS Ch., 1893 - « Notes sur quelques nécropoles mégalithiques du centre de la Tunisie », BAC, p. 138-143.

M'CHAREK A., 1988 - « Maghrawa, lieu de provenance des stèles punico-numides dites de la Ghorfa », MEFRA, 100-2, p. 731-760.

M'CHAREK A., 1990-91 - « Maghrawa, antique Macota au I ${ }^{\mathrm{er}}$ siècle apr. J.-C. » (résumé), BCTH, 1991 (P. v. du 16 décembre) et Bulletin des travaux de l'INP, Tunis, $\mathrm{n}^{\circ}$ 6, p. 75-78.

M'CHAREK A., 1997 - « Maghrawa, antique Macota (Tunisie) », Antiquités Africaines, t. 33, p. 119-127.

\section{INDEX}

Mots-clés : Antiquité, Epigraphie, Libyque, Monument funéraire, Protohistoire, Punique, Tunisie 